\title{
Lieferengpässe behindern Produktion
}

Seit dem Beginn der Corona-Pandemie führen Lieferengpässe zu spürbaren Produktionsbehinderungen bei Unternehmen in der Industrie. Nachdem die Engpässe mit dem Überwinden der ersten Pandemie-Welle offenbar zunächst nachgelassen hatten, haben die Belastungen zuletzt wieder merklich zugenommen. So berichteten im April mehr als $40 \%$ der Unternehmen im Verarbeitenden Gewerbe in Deutschland von Produktionsstörungen aufgrund eines Mangels an Rohstoffen bzw. Vorleistungen der höchste jemals verzeichnete Wert im wiedervereinigten Deutschland (vgl. Abbildung 1). Während die Lieferengpässe zu Beginn der Pandemie im Frühjahr 2020 wohl im Wesentlichen auf Grenzschließungen in Verbindung mit anderen Infektionsschutzmaßnahmen zurückzuführen waren, trafen danach mehrere andere belastende Faktoren zusammen. So hat die auf internationaler Ebene zeitlich heterogene wirtschaftliche Erholung - ausgehend von der früh einsetzenden Erholung in China im vergangenen Jahr - zu einer Unwucht im Schifffahrtsverkehr geführt, die offenbar bis zuletzt angehalten hat und spürbare Transportengpässe mit sich bringt. Dazu beigetragen hat auch die pandemiebedingte Verlagerung der privaten

(C) Der/die Autor:in(nen) 2021. Open Access: Dieser Artikel wird unter der Creative Commons Namensnennung 4.0 International Lizenz veröffentlicht (creativecommons.org/licenses/by/4.0/deed.de).

Open Access wird durch die ZBW - Leibniz-Informationszentrum Wirtschaft gefördert.

\section{Abbildung 1}

Mangel an Rohstoffen/Vormaterialien im

Verarbeitenden Gewerbe

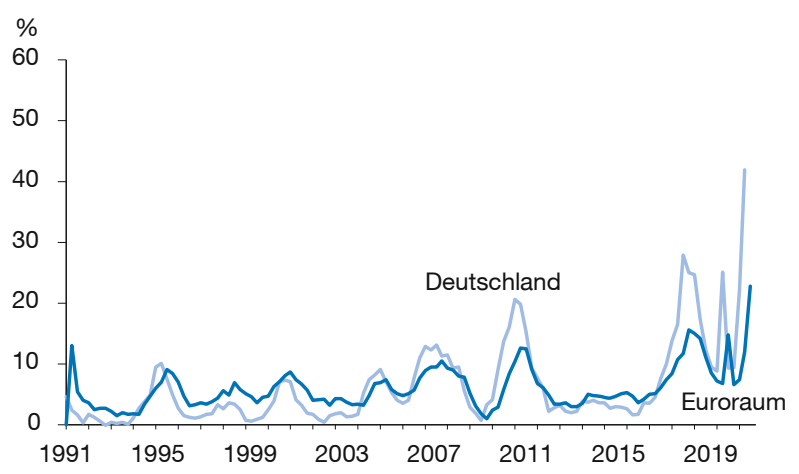

Anmerkungen: Quartalsdaten, saisonbereinigt. Anteil der Unternehmen die angeben, dass ihre Produktion durch einen Mangel an Rohstoffen oder Vorleistungen behindert wird.

Quelle: Europäische Kommission.
Konsumausgaben von Dienstleistungen zu langlebigen Konsumgütern. Durch diese Verlagerung zog der Bedarf an elektronischen Vorleistungsgütern, wie Halbleitern, kräftig an und traf dabei auf ein offenbar kurzfristig recht starres Angebot. Schließlich dürften viele Unternehmen bei ihren Produktionsdispositionen von der Stärke der wirtschaftlichen Erholung, die deutlich kräftiger ausfiel als im Anschluss an frühere Krisen, überrascht worden sein. Hinzu kamen einzelne Ereignisse (z. B. die vorübergehende Blockade des Suezkanals, Corona-Ausbrüche in großen Häfen oder Brände in Chip-Fabriken), welche die bereits angespannten Lieferketten zusätzlich belastet haben. Unternehmensbefragungen zufolge haben diese Lieferengpässe die Produktion in Deutschland stärker behindert als in vielen anderen europäischen Volkswirtschaften. So lag der Anteil der Unternehmen im Verarbeitenden Gewerbe, die von einem Rohstoff- bzw. Vorleistungsmangel berichteten, im Euroraum insgesamt mit $23 \%$ zwar ebenfalls auf erhöhtem Niveau, jedoch deutlich niedriger als in Deutschland. Dazu beigetragen haben dürfte, dass die Knappheit bei elektronischen Bauteilen offenbar besonders bei den Automobilherstellern zu Buche geschlagen hat und diese Branche für die deutsche Wirtschaft recht bedeutsam ist (Jannsen, 2019).

Die Auswirkungen der Lieferengpässe auf die Produktion lassen sich nicht direkt beobachten. Auffällig ist jedoch, dass die Erholung in der Industrie trotz guter Auftragslage seit Jahresbeginn ins Stocken geraten ist. So ging die Industrieproduktion in den ersten Monaten 2021 sogar zurück und lag im Mai noch unterhalb ihres Vorkrisenniveaus. Demgegenüber legten die Auftragseingänge in der Tendenz weiter zu und befinden sich bereits seit mehreren Monaten deutlich oberhalb ihres Vorkrisenniveaus. Angesichts der Unternehmensmeldungen zu den Produktionshemmnissen ist es naheliegend, dass die Lieferengpässe wesentlich zu dieser Diskrepanz beigetragen haben. Vor diesem Hintergrund lassen sich die Auswirkungen der Lieferengpässe quantitativ eingrenzen, indem man auf Basis des langfristigen Zusammenhangs zwischen Auftragseingängen und Industrieproduktion ein „normales" Niveau der Produktion abschätzt, das angesichts der gegenwärtigen Auftragslage zu erwarten wäre. Abweichungen von einem solchen Referenzwert, die auch zuvor vorübergehend immer wieder zu verzeichnen waren, können dann für die jüngere Vergangenheit als Einfluss der Lieferengpässe interpretiert werden. Empirische Analysen deuten auf einen robusten langfristigen Zusam- 


\section{Abbildung 2}

Tatsächliche Produktion im Verarbeitenden Gewerbe und Langfristbeziehung mit den Auftragseingängen

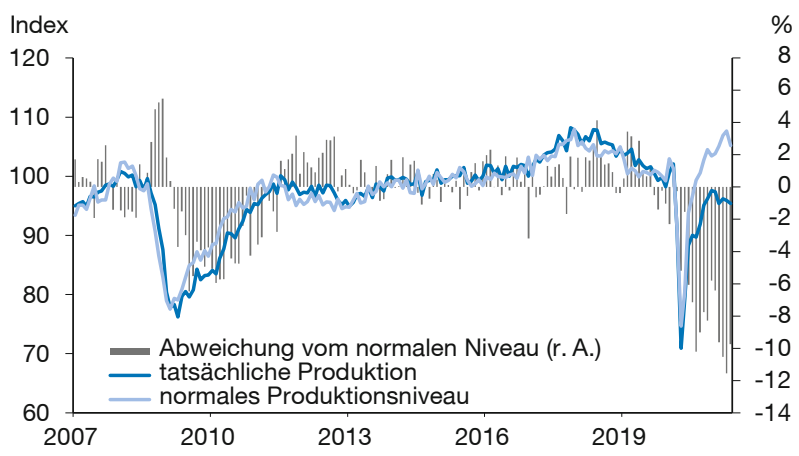

Anmerkungen: Monatsdaten, saison- und kalenderbereinigt. Normales Produktionsniveau geschätzt mittels einer Kointegrationsbeziehung zwischen Auftragseingängen und Produktion.

Quelle: Deutsche Bundesbank, Saisonbereinigte Wirtschaftszahlen; Berechnungen des IfW Kiel.

menhang zwischen Auftragseingängen und Industrieproduktion hin. ${ }^{1}$ Seit dem Beginn der Corona-Pandemie ist die Produktion demnach deutlich hinter ihrem „normalen“ Niveau zurückgeblieben. Zuletzt lag die Produktion sogar um rund $10 \%$ unterhalb des zu erwarteten Niveaus; eine so hohe Abweichung war vor der Corona-Krise noch nie verzeichnet worden (vgl. Abbildung 2). Freilich dürfte das niedrige Produktionsniveau nicht ausschließlich auf Lieferengpässe zurückzuführen sein. So wurde die Produktion insbesondere zu Beginn der Pandemie im Frühjahr 2020 durch Infektionsschutzmaßnahmen behindert. Auch ist es nicht ungewöhnlich, dass zu Beginn von Erholungsphasen die Auftragseingänge zunächst stärker anziehen als die Produktion, so wie es beispielsweise im Anschluss an die globale Finanzkrise zu beobachten war. Auch im August 2020, während der wirtschaftlichen Erholung von der ersten Pandemie-Welle, blieb die Produktion zwischenzeitlich $10 \%$ unterhalb des zu erwartenden Niveaus zurück. Bis zum Jahresende konnte dieser Produktionsunterhang um rund die Hälfte wieder abgebaut werden. Seit Jahresbeginn stieg der Abstand jedoch wieder um rund 5 Prozentpunkte an.

Geht man davon aus, dass die schwache Produktion im Verarbeitenden Gewerbe seit Jahresbeginn auf die Lieferengpässe zurückzuführen ist, so ist dadurch die Pro-

1 In den empirischen Analysen wird der langfristige Zusammenhang als Kointegrationsbeziehung durch Verfahren von Engle und Granger bzw. Johannsen modelliert. Die Schätzungen wurden auf Basis monatlicher Daten für unterschiedliche Zeiträume ab 1991 vorgenommen. Als Basisspezifikation dient eine Schätzung bis Ende 2019. Die Schätzungen deuten auf eine robuste Kointegrationsbeziehung hin, sofern für einzelne Strukturbrüche im Schätzzeitraum kontrolliert wird (Beckmann und Jannsen, 2021). duktion allein im ersten Quartal (gegenüber dem vierten Quartal 2020) um etwa 1,5\% gedrückt worden. Für das zweite Quartal zeichnet sich eine zusätzliche Belastung von $1,5 \%$ ab, sodass das Produktionsniveau dann um etwa $3 \%$ unterhalb des Niveaus liegen würde, das ohne die Lieferengpässe seit Jahresbeginn zu erwarten gewesen wäre. Bei einem Anteil des Verarbeitenden Gewerbes von rund $20 \%$ an der gesamten Bruttowertschöpfung in Deutschland ergeben sich somit auch gesamtwirtschaftlich spürbare Auswirkungen.

Freilich handelt es sich bei den Lieferengpässen im Kern um ein temporäres Phänomen, durch das die Produktion lediglich verzögert wird. Sobald sich die Engpässe lösen, wird die Produktion wieder spürbar anziehen, sofern es zwischenzeitlich nicht zu Stornierungen gekommen ist oder die Belastungen durch die Lieferengpässe ihrerseits nicht zu geringeren Auftragseingängen geführt haben werden. Die Produktion erhält dann einen doppelten Schub. Erstens wird sich die Produktion wieder ihrem „normalen" Niveau annähern. Allein durch diesen Aufholeffekt würde die Produktion dann um rund $10 \%$ stärker zulegen als die Auftragseingänge. Zweitens werden die zuvor aufgelaufenen Auftragseingänge dann von den Unternehmen möglichst rasch wieder abgearbeitet werden. Den Schätzergebnissen zufolge konnten allein zwischen Januar und Mai Auftragseingänge im Umfang von etwa $50 \%$ einer Monatsproduktion nicht abgearbeitet werden. Durch den resultierenden Nachholeffekt würde die Produktion vorübergehend über ihr "normales“ Niveau hinausgehen. Wann die Lieferengpässe die Produktion nicht mehr belasten werden, ist auch aufgrund der Vielschichtigkeit der zugrunde liegenden Ursachen schwer absehbar. Derzeit deutet die Nachrichtenlage eher darauf hin, dass die Engpässe noch für einige Monate anhalten werden. In einem Szenario, in dem sich die Lieferengpässe nach und nach bis zum Jahresende auflösen, würde die Produktion in diesem Zeitraum zwar stärker zulegen als die Auftragseingänge. Jedoch würde die Produktion dann vorerst weiterhin unter ihrem „normalen“ Niveau bleiben und sich die Auftragsbestände somit weiter erhöhen.

Joscha Beckmann, Nils Jannsen Joscha.Beckmann@ifw-kiel.de, Nils.Jannsen@ifw-kiel.de

\section{Literatur}

Beckmann, J. und N. Jannsen (2021), Bedeutung von Lieferengpässen für die laufende Produktion in Deutschland, IfW-Box, 2021.09, https:// www.ifw-kiel.de/fileadmin/Dateiverwaltung/IfW-Publications/-ifw/ IfW_Box/2021/Box_2021-9_Deutschland_Sommer.pdf (5. Juli 2021).

Jannsen, N. (2019), Zur Bedeutung der Automobilindustrie für die deutsche Wirtschaft, Wirtschaftsdienst, 99(7), 451-456, https://www.wirtschaftsdienst.eu/inhalt/jahr/2019/heft/7/beitrag/autoindustrie-aufdem-richtigen-weg.html (5. Juli 2021). 